\title{
New Aspects of ZZ Transform to Fractional Operators With Mittag-Leffler Kernel
}

\author{
Rajarama Mohan Jena ${ }^{1}$, Snehashish Chakraverty ${ }^{1 *}$, Dumitru Baleanu ${ }^{2,3}$ and \\ Maysaa M. Alqurashi ${ }^{4}$
}

${ }^{1}$ Department of Mathematics, National Institute of Technology Rourkela, Rourkela, India, ${ }^{2}$ Department of Mathematics, Faculty of Art and Sciences, Cankaya University Balgat, Ankara, Turkey, ${ }^{3}$ Institute of Space Sciences, Magurele-Bucharest, Romania, ${ }^{4}$ Department of Mathematics, King Saud University, Riyadh, Saudi Arabia

In this paper, we discuss the relationship between the Zain UI Abadin Zafar (ZZ) transform with Laplace and Aboodh transforms. Further, the ZZ transform is applied to the fractional derivative with the Mittag-Leffler kernel defined in both the Caputo and Riemann-Liouville sense. In order to illustrate the validity and applicability of the transform, we solve some illustrative examples.

\section{OPEN ACCESS}

Edited by:

Jordan Yankov Hristov,

University of Chemical Technology and Metallurgy, Bulgaria

Reviewed by:

Praveen Agarwal,

Anand International College of

Engineering, India

Yang Liu,

Inner Mongolia University, China

Shilpi Jain

Poornima College of Engineering, India

${ }^{*}$ Correspondence:

Snehashish Chakraverty

sne_chak@yahoo.com

Specialty section:

This article was submitted to Mathematical and Statistical Physics,

a section of the journal

Frontiers in Physics

Received: 25 February 2020

Accepted: 24 July 2020

Published: 23 September 2020

Citation:

Jena RM, Chakraverty S, Baleanu D

and Alqurashi MM (2020) New

Aspects of ZZ Transform to Fractional

Operators With Mittag-Leffler Kernel.

Front. Phys. 8:352.

doi: 10.3389/fphy.2020.00352

Keywords: ZZ transform, fractional calculus, aboodh transform, non-singular kernel, mittag-leffler kernel

\section{OUTLINE AND MOTIVATION}

In recent years, fractional calculus (FC) has gained considerable achievements in various fields of science and engineering. Many physical problems [1-7] are modeled by using fractional differential equations (FDE) more accurately than classical differential equations [8-11]. Earlier, various real-life problems were modeled by using the Caputo and Riemann-Liouville (R-L) fractional derivatives. However, Caputo and Fabrizio proposed a new idea that reflects the exponential kernel [12] to address a new way of modeling phenomena with non-local effects. Further, in [13], a new fractional operator $(\mathrm{AB})$ with a Mittag-Leffler kernel was developed. So, in this regard, many researchers [14-16] have given their interest in this definition to solve various problems/models. In fact, in modeling real phenomena, we need a variety of fractional operators to thoroughly describe the complexity of the problem studied. Some other studies regarding fractional calculus and special functions can be found in the literature [17-26].

In the present study, we establish the relationship between the ZZ transform (ZZT) with the Aboodh transform (AT), and the Laplace transform (LT) having their various applications given in [27-31]. Next, the ZZT has been applied to AB fractional operators defined in the Caputo and R-L sense, which are described in terms of theorems. Later, we have solved some test examples defined in the AB sense using this ZZT. The contribution of the present authors to this manuscript are (i) firstly establishing the relationship among ZZT, LT, and AT, (ii) secondly applying ZZT to fractional differential equations defined in the $\mathrm{AB}$ derivative to get the solution of the problems. The $\mathrm{ZZ}$ transform is the generalization of some famous transforms and we can relate this transformation to other well-known transforms. If we divide the $\mathrm{ZZ}$ transform by the transformed variable, then we get the Natural transform. Similarly, relations with other integral transforms in terms of theorems have been included in this paper. The main benefit of this transformation is that it may converge to the Sumudu transform and is advantageous in solving FDEs with variable coefficients.

The organization of the paper is as follows: In section Preliminaries and Basic Definitions, we establish the connection between the Aboodh and ZZ transform; we prove some significant results and create the relationships between AB derivatives with ZZT. In section Applications, some FDEs are solved using ZZT. Finally, a conclusion section is included in section Conclusion. 


\section{PRELIMINARIES AND BASIC DEFINITIONS}

\section{Definition 2.1}

The Aboodh transform is obtained on the set of functions

$$
B=\left\{f(t): \exists M, m_{1}, m_{2}>0,|f(t)|<M e^{-s t}\right\}
$$

and is defined as $[27,28]$

$$
A\{f(t)\}=\frac{1}{s} \int_{0}^{\infty} f(t) e^{-s t} d t, t>0 \text { and } m_{1} \leq s \leq m_{2}
$$

\section{Theorem 2.1}

Let us consider $G$ and $F$ as the Aboodh and Laplace transforms of $f(t) \in B$ then [32]

$$
G(s)=\frac{F(s)}{s} .
$$

The ZZT was introduced by Zain Ul Abadin Zafar [29, 30]. It generalizes the Aboodh and Laplace integral transforms. In the following definition, we discuss the definition of ZZT.

\section{Definition 2.2 (ZZ Transform)}

Suppose $f(t) \forall t \geq 0$ is a function then the ZZT $Z(v, s)$ of $f(t)$ is defined as $[29,30]$

$$
Z Z(f(t))=Z(v, s)=s \int_{0}^{\infty} f(v t) e^{-s t} d t
$$

Similar to the Aboodh and Laplace transforms, the ZZT is also linear. The MLF is an extension of exponential function which is defined as.

$$
E_{\alpha}(z)=\sum_{n=0}^{\infty} \frac{z^{n}}{\Gamma(1+n \alpha)}, \quad \operatorname{Re}(\alpha)>0 .
$$

\section{Definition 2.3}

Let us consider a function $\xi(x, t) \in H^{1}(a, b)$, then for $\alpha \in$ $(0,1)$, the Atangana-Baleanu Caputo $(\mathrm{ABC})$ derivative is written as [13].

$$
A B C{ }_{a} D_{t}^{\alpha} \xi(x, t)=\frac{\psi(\alpha)}{1-\alpha} \int_{a}^{t} \xi^{\prime}(x, \tau) E_{\alpha}\left(\frac{-\alpha(t-\tau)^{\alpha}}{1-\alpha}\right) d \tau .
$$

\section{Definition 2.4}

Let $\xi(x, t) \in H^{1}(a, b)$, then for $\alpha \in(0,1)$, the Atangana-Baleanu Riemann-Liouville (ABR) derivative is given as [13]

$$
{ }_{a}^{A B R} D_{t}^{\alpha} \xi(x, t)=\frac{\psi(\alpha)}{1-\alpha} \frac{d}{d t} \int_{a}^{t} \xi(x, \tau) E_{\alpha}\left(\frac{-\alpha(t-\tau)^{\alpha}}{1-\alpha}\right) d \tau,
$$

where $\psi(\alpha)$ is a function with the conditions $\psi(0)=\psi(1)=$ land $b>a$.

\section{Theorem 2.2}

The LT of $\mathrm{ABC}$ and $\mathrm{ABR}$ derivative are, respectively, given as [13]

$$
\mathrm{L}\left\{{ }_{a}^{A B C} D_{t}^{\alpha} \xi(x, t)\right\}(s)=\frac{\psi(\alpha)}{1-\alpha} \frac{s^{\alpha} L\{\xi(x, t)\}-s^{\alpha-1} \xi(x, 0)}{s^{\alpha}+\frac{\alpha}{1-\alpha}}
$$

and

$$
\mathrm{L}\left\{{ }_{a}^{A B R} D_{t}^{\alpha} \xi(x, t)\right\}(s)=\frac{\psi(\alpha)}{1-\alpha} \frac{s^{\alpha} L\{\xi(x, t)\}}{s^{\alpha}+\frac{\alpha}{1-\alpha}} .
$$

The following theorems have been proposed where it is assumed that $f(t) \in H^{1}(a, b), b>$ and $\alpha \in(0,1)$.

\section{Theorem 2.3}

The AT of ABC derivative is given as.

$$
\begin{aligned}
G(s)= & A\left\{{ }_{a}^{A B C} D_{t}^{\alpha} \xi(x, t)\right\}(s) \\
= & \frac{1}{s}\left[\frac{\psi(\alpha)}{1-\alpha} \frac{s^{\alpha} L\{\xi(x, t)\}-s^{\alpha-1} \xi(x, 0)}{s^{\alpha}+\frac{\alpha}{1-\alpha}}\right] .
\end{aligned}
$$

Proof: Using Theorem 2.1 and Equation. (2.2), we may get the desired result.

\section{Theorem 2.4}

The Aboodh transform of ABR derivative is written as.

$$
G(s)=A\left\{{ }_{a}^{A B R} D_{t}^{\alpha} \xi(x, t)\right\}(s)=\frac{1}{s}\left[\frac{\psi(\alpha)}{1-\alpha} \frac{s^{\alpha} L\{\xi(x, t)\}}{s^{\alpha}+\frac{\alpha}{1-\alpha}}\right] .
$$

\section{Proof}

Applying the Theorem 2.1 and Equation (2.3), we obtain the required result.

The connection between the transforms of Aboodh and $\mathrm{ZZ}$ is given in the theorem below.

\section{Theorem 2.5}

If $G(s)$ and $Z(v, s)$ are the Aboodh and ZZ transforms of $f(t) \in B$. Then, we obtain

$$
Z(v, s)=\frac{s^{2}}{v^{2}} G\left(\frac{s}{v}\right)
$$

Proof. From the definition of $\mathrm{ZZ}$ transform we have

$$
Z(v, s)=s \int_{0}^{\infty} f(v t) e^{-s t} d t
$$


Substituting $v t=\tau$ in Equation (2.6) we get

$$
Z(v, s)=\frac{s}{v} \int_{0}^{\infty} f(\tau) e^{-\frac{s \tau}{v}} d \tau
$$

The right-hand side of the above Equation (2.7) may be written as.

$$
Z(v, s)=\frac{s}{v} F\left(\frac{s}{v}\right)
$$

where $F($.$) denotes the Laplace transform of f(t)$.

Applying the Theorem 2.1, Equation (2.8) can be expressed as

$$
Z(v, s)=\frac{s}{v} \frac{F\left(\frac{s}{v}\right)}{\left(\frac{s}{v}\right)} \times\left(\frac{s}{v}\right)=\left(\frac{s}{v}\right)^{2} G\left(\frac{s}{v}\right)
$$

where $G($.) denotes the Aboodh transform of $f(t)$.

\section{Theorem 2.6}

$\mathrm{ZZ}$ transform of $f(t)=t^{\alpha-1}$ is given as

$$
Z(v, s)=\Gamma(\alpha)\left(\frac{v}{s}\right)^{\alpha-1}
$$

Proof. The Aboodh transform of $f(t)=t^{\alpha}, \alpha \geq 0$ is

$$
G(s)=\frac{\Gamma(\alpha)}{s^{\alpha+1}}
$$

Now, $\quad G\left(\frac{s}{v}\right)=\frac{\Gamma(\alpha) v^{\alpha+1}}{s^{\alpha+1}}$.

Using Equation (2.9), we obtain.

$$
Z(v, s)=\frac{s^{2}}{v^{2}} G\left(\frac{s}{v}\right)=\frac{s^{2}}{v^{2}} \frac{\Gamma(\alpha) v^{\alpha+1}}{s^{\alpha+1}}=\Gamma(\alpha)\left(\frac{v}{s}\right)^{\alpha-1}
$$

\section{Theorem 2.7}

Let $\alpha, \omega \in C$ and $\operatorname{Re}(\alpha)>0$, then the ZZ transform of $E_{\alpha}\left(\omega t^{\alpha}\right)$ is given as

$$
Z Z\left\{\left(E_{\alpha}\left(\omega t^{\alpha}\right)\right)\right\}=Z(v, s)=\left(1-\omega\left(\frac{v}{s}\right)^{\alpha}\right)^{-1}
$$

Proof. We know that Aboodh transform of $E_{\alpha}\left(\omega t^{\alpha}\right)$ is written as.

$$
\begin{gathered}
G(s)=\frac{F(s)}{s}=\frac{s^{\alpha-1}}{s\left(s^{\alpha}-\omega\right)}, \\
\text { So, } G\left(\frac{s}{v}\right)=\frac{\left(\frac{s}{v}\right)^{\alpha-1}}{\left(\frac{s}{v}\right)\left(\left(\frac{s}{v}\right)^{\alpha}-\omega\right)},
\end{gathered}
$$

Using the Theorem 2.9, we obtain.

$$
\begin{aligned}
Z(v, s)= & \left(\frac{s}{v}\right)^{2} G\left(\frac{s}{v}\right)=\left(\frac{s}{v}\right)^{2} \frac{\left(\frac{s}{v}\right)^{\alpha-1}}{\left(\frac{s}{v}\right)\left(\left(\frac{s}{v}\right)^{\alpha}-\omega\right)} \\
= & \frac{\left(\frac{s}{v}\right)^{\alpha}}{\left(\frac{s}{v}\right)^{\alpha}-\omega}=\left(1-\omega\left(\frac{v}{s}\right)^{\alpha}\right)^{-1} .
\end{aligned}
$$

\section{Theorem 2.8}

If $G(s)$ and $Z(v, s)$ are the Aboodh and ZZ transforms of $f(t)$.

Then the $\mathrm{ZZT}$ of $\mathrm{ABC}$ derivative is written as.

$$
Z Z\left\{\begin{array}{l}
{ }_{0} B C \\
0
\end{array} D_{t}^{\alpha} f(t)\right\}=\left[\frac{\psi(\alpha)}{1-\alpha} \frac{\frac{s^{\alpha+2}}{v^{\alpha+2}} G\left(\frac{s}{v}\right)-\frac{s^{\alpha}}{v^{\alpha}} f(0)}{\frac{s^{\alpha}}{v^{\alpha}}+\frac{\alpha}{1-\alpha}}\right] .
$$

Proof. Using the Equations (2.1) and (2.4), we have

$$
G\left(\frac{s}{v}\right)==\frac{v}{s}\left[\frac{\psi(\alpha)}{1-\alpha} \frac{\left(\frac{s}{v}\right)^{\alpha+1} G\left(\frac{s}{v}\right)-\left(\frac{s}{v}\right)^{\alpha-1} f(0)}{\left(\frac{s}{v}\right)^{\alpha}+\frac{\alpha}{1-\alpha}}\right] .
$$

So, the $\mathrm{ZZ}$ transform of $\mathrm{ABC}$ is given as.

$$
\begin{aligned}
Z(v, s)= & \left(\frac{s}{v}\right)^{2} G\left(\frac{s}{v}\right) \\
& =\left(\frac{s}{v}\right)^{2} \frac{v}{s}\left[\frac{\psi(\alpha)}{1-\alpha} \frac{\left(\frac{s}{v}\right)^{\alpha+1} G\left(\frac{s}{v}\right)-\left(\frac{s}{v}\right)^{\alpha-1} f(0)}{\left(\frac{s}{v}\right)^{\alpha}+\frac{\alpha}{1-\alpha}}\right] \\
& =\left[\frac{\psi(\alpha)}{1-\alpha} \frac{\left(\frac{s}{v}\right)^{\alpha+2} G\left(\frac{s}{v}\right)-\left(\frac{s}{v}\right)^{\alpha} f(0)}{\left(\frac{s}{v}\right)^{\alpha}+\frac{\alpha}{1-\alpha}}\right]
\end{aligned}
$$

\section{Theorem 2.9}

Let us assume that $G(s)$ and $Z(v, s)$ are the Aboodh and $Z Z$ transform of $f(t)$. Then the ZZ transform of ABR derivative is given as

$$
Z Z\left\{{ }_{0}^{A B R} D_{t}^{\alpha} f(t)\right\}=\left[\frac{\psi(\alpha)}{1-\alpha} \frac{\frac{s^{\alpha+2}}{v^{\alpha+2}} G\left(\frac{s}{v}\right)}{\frac{s^{\alpha}}{v^{\alpha}}+\frac{\alpha}{1-\alpha}}\right] .
$$

Proof. Using the Equations (2.1) and (2.5), we get

$$
G\left(\frac{s}{v}\right)=\frac{v}{s}\left[\frac{\psi(\alpha)}{1-\alpha} \frac{\left(\frac{s}{v}\right)^{\alpha+1} G\left(\frac{s}{v}\right)}{\left(\frac{s}{v}\right)^{\alpha}+\frac{\alpha}{1-\alpha}}\right] .
$$

From the Equation (2.9), the $\mathrm{ZZ}$ transform of $\mathrm{ABR}$ is written as.

$$
\begin{array}{r}
Z(v, s)=\left(\frac{s}{v}\right)^{2} G\left(\frac{s}{v}\right)=\left(\frac{s}{v}\right)^{2}\left(\frac{v}{s}\right)\left[\frac{\psi(\alpha)}{1-\alpha} \frac{\left(\frac{s}{v}\right)^{\alpha+1} G\left(\frac{s}{v}\right)}{\left(\frac{s}{v}\right)^{\alpha}+\frac{\alpha}{1-\alpha}}\right] \\
=\left[\frac{\psi(\alpha)}{1-\alpha} \frac{\left(\frac{s}{v}\right)^{\alpha+2} G\left(\frac{s}{v}\right)}{\left(\frac{s}{v}\right)^{\alpha}+\frac{\alpha}{1-\alpha}}\right] .
\end{array}
$$




\section{APPLICATIONS}

Let us consider the following initial value problem (IVP) defined in $\mathrm{ABC}$ sense [15]

$$
\left\{\begin{array}{l}
{ }^{A B C} D_{t}^{\alpha} y(t)=f(t, y(t)), t>0 \\
y(0)=k, \quad k \in \Re
\end{array}\right.
$$

Suppose $Z(v, s)$ and $T(v, s)$ are the ZZ transforms of $y(t)$ and $f$, respectively. Then by taking the ZZT on both sides of Equation (3.1) and using Equations (2.9) and (2.14), we may get

$$
\begin{gathered}
{\left[\frac{\psi(\alpha)}{1-\alpha} \frac{\left(\frac{s}{v}\right)^{\alpha} Z(v, s)-\left(\frac{s}{v}\right)^{\alpha} y(0)}{\left(\frac{s}{v}\right)^{\alpha}+\frac{\alpha}{1-\alpha}}\right]=T(v, s) .} \\
{\left[\psi(\alpha) \frac{Z(v, s)-k}{\left(1-\alpha+\alpha\left(\frac{v}{s}\right)^{\alpha}\right)}\right]=T(v, s)} \\
\text { Thus, } Z(v, s)=\frac{1-\alpha+\alpha\left(\frac{v}{s}\right)^{\alpha}}{\psi(\alpha)} T(v, s)+k .
\end{gathered}
$$

Then, by applying the inverse ZZT on both sides of Equation (3.2), we obtain the exact solution.

Similarly, we may solve Equation (3.1) defined in ABR derivative.

\section{Example 3.1}

Let us take the following fractional IVP [15]

$$
\left\{\begin{array}{l}
{ }^{A B C} D_{t}^{\alpha} y(t)=y(t), t>0 \\
y(0)=1
\end{array}\right.
$$

Firstly, we apply the ZZT on both sides of Equation (3.3) which gives

$$
\left[\frac{\psi(\alpha)}{1-\alpha} \frac{\left(\frac{s}{v}\right)^{\alpha} Z(v, s)-\left(\frac{s}{v}\right)^{\alpha} y(0)}{\left(\frac{s}{v}\right)^{\alpha}+\frac{\alpha}{1-\alpha}}\right]=Z(v, s) .
$$

Simplifying Equation (3.4) and using the initial condition, we have

$$
\left[\psi(\alpha) \frac{Z(v, s)-1}{\left(1-\alpha+\alpha\left(\frac{v}{s}\right)^{\alpha}\right)}\right]=Z(v, s) .
$$

The simplification of Equation (3.5) gives us the following:

$$
Z(v, s)=\frac{1}{1-\frac{1-\alpha+\alpha\left(\frac{v}{s}\right)^{\alpha}}{\psi(\alpha)}}=\frac{\psi(\alpha)}{\psi(\alpha)-1+\alpha-\alpha\left(\frac{v}{s}\right)^{\alpha}} .
$$

Equation (3.6) may be rewritten as.

$$
Z(v, s)=\frac{\psi(\alpha)}{(\psi(\alpha)-1+\alpha)}\left(1-\frac{\alpha}{\psi(\alpha)-1+\alpha}\left(\frac{v}{s}\right)^{\alpha}\right)^{-1}
$$

Applying the inverse of the ZZT on Equation (3.7) and using Equation (2.11), Equation (3.7) is reduced to

$$
y(t)=\frac{\psi(\alpha)}{(\psi(\alpha)-1+\alpha)} E_{\alpha}\left(\frac{\alpha}{\psi(\alpha)-1+\alpha} t^{\alpha}\right),
$$

where $E_{\alpha}(t)$ is the MLF.

Substituting $\alpha=1$ in Equation (3.8), we obtain

$$
y(t)=E_{1}(t)=e^{t}
$$

which is the exact solution of Equation (3.3) when $\alpha=1$.

\section{Example 3.2}

Considering the following fractional IVP [15]

$$
\left\{\begin{array}{l}
{ }^{A B C} D_{t}^{\alpha} y(t)=\eta t, t>0 \\
y(0)=0
\end{array}\right.
$$

Taking the ZZT on both sides of Equation (3.10) and plugging the initial condition, we get

$$
\left[\frac{\psi(\alpha)}{1-\alpha} \frac{\left(\frac{s}{v}\right)^{\alpha} Z(v, s)-\left(\frac{s}{v}\right)^{\alpha} y(0)}{\left(\frac{s}{v}\right)^{\alpha}+\frac{\alpha}{1-\alpha}}\right]=\eta\left(\frac{v}{s}\right)
$$

$$
\left[\psi(\alpha) \frac{Z(v, s)}{\left(1-\alpha+\alpha\left(\frac{v}{s}\right)^{\alpha}\right)}\right]=\eta\left(\frac{v}{s}\right)
$$

$$
\begin{aligned}
Z(v, s)= & \eta\left(\frac{v}{s}\right) \frac{\left(1-\alpha+\alpha\left(\frac{v}{s}\right)^{\alpha}\right)}{\psi(\alpha)} \\
& =\frac{\eta}{\psi(\alpha)}\left[(1-\alpha)\left(\frac{v}{s}\right)+\alpha\left(\frac{v}{s}\right)^{\alpha+1}\right]
\end{aligned}
$$

Applying inverse ZZT on both sides of Equation (3.11), we obtain

$$
y(t)=\frac{\eta}{\psi(\alpha)}\left[(1-\alpha) t+\frac{\alpha}{\Gamma(\alpha+2)} t^{\alpha+1}\right] .
$$

It is noticed that if we put $\alpha=0$, then Equation (3.12) reduces to $y(t)=\eta t$ and substituting $\alpha=1$ in Equation (3.12), we obtain $y(t)=\eta \frac{t^{2}}{2}$. Plugging $\alpha=0.5$, we get $y(t)=$ $\frac{\eta}{\psi(0.5)}\left[\frac{t}{2}+\frac{2}{3 \sqrt{\pi}} t^{\frac{3}{2}}\right]$.

\section{CONCLUSION}

In this manuscript, the ZZT is debated and the associated properties of ZZT are established. Some theorems related to the connection between the ZZ, Aboodh, and Laplace transforms are successfully proven. ZZT was applied to FDEs within the $\mathrm{AB}$ derivatives. Besides, some fractional initial value problems are solved in order to illustrate the validity and performance of this transformation. 


\section{DATA AVAILABILITY STATEMENT}

The original contributions presented in the study are included in the article/supplementary materials, further inquiries can be directed to the corresponding author/s.

\section{AUTHOR CONTRIBUTIONS}

RJ: conceptualization, writing-original draft, methodology, software, and validation. SC and DB: project administration and supervision. MA and DB: funding acquisition. SC, DB, and MA:

\section{REFERENCES}

1. Jena RM, Chakraverty S. Residual power series method for solving time-fractional model of vibration equation of large membranes. $J$ Appl Comput Mech. (2019) 5:603-15. doi: 10.22055/jacm.2018.2666 8.1347

2. Jena RM, Chakraverty S. A new iterative method based solution for fractional black-scholes option pricing equations (BSOPE). SN Appl Sci. (2019) 1:95. doi: 10.1007/s42452-018-0106-8

3. Jena RM, Chakraverty S, Jena SK. Dynamic response analysis of fractionally damped beams subjected to external loads using homotopy analysis method (HAM). J Appl Comput Mech. (2019) 5:355-66. doi: 10.22055/JACM.2019.2759 2.1419

4. Edeki SO, Akinlabi GO, Jena RM, Ogundile OP. Conformable decomposition method for time-space fractional intermediate scalar transportation model. $J$ Theor Appl Inform Technol. (2019) 97:4251-8.

5. Jena RM, Chakraverty S, Baleanu D. On the solution of imprecisely defined nonlinear time-fractional dynamical model of marriage. Mathematics. (2019) 7:689. doi: 10.3390/math7 080689

6. Jena RM, Chakraverty S, Baleanu D. On new solutions of time-fractional wave equations arising in shallow water wave propagation. Mathematics. (2019) 7:722. doi: 10.3390/math70 80722

7. Jena RM, Chakraverty S. Boundary characteristic orthogonal polynomials-based galerkin and least square methods for solving bagley-torvik equations. Recent Trends Wave Mech Vibrations. (2019) 13:327-42. doi: 10.1007/978-981-15-02 87-3_24

8. Baleanu D, Machado JAT, Luo ACJ. Fractional Dynamics, and Control. New York, NY: Springer (2012). doi: 10.1007/978-1-4614-0457-6

9. Baleanu D, Diethelm K, Scalas E, Trujillo JJ. Fractional Calculus: Models and Numerical Methods. Singapore: World Scientific Publishing Company (2012). doi: 10.1142/8180

10. Kilbas AA, Srivastava HM, Trujillo JJ. Theory and Application of Fractional Differential Equations. Amsterdam: Elsevier Science B.V (2006).

11. Yang XJ, Baleanu D, Srivastava HM. Local Fractional Integral Transform and their Applications. New York, NY: Academic Press (2015). doi: 10.1016/B978-0-12-804002-7. 00004-8

12. Caputo M, Fabrizio M. A new definition of fractional derivative without singular kernel. Progr Fract Differ Appl. (2015) 1:113. doi: $10.18576 /$ pfda/020101

13. Atangana A, Baleanu D. New fractional derivatives with nonlocal and non-singular kernel: theory and application to heat transfer model. Therm Sci. (2016) 20:763-9. doi: 10.2298/TSCI1601 $11018 \mathrm{~A}$

14. Saad KM, Atangana A, Baleanu D. New fractional derivatives with non-singular kernel applied to the burgers equation. Chaos contributed to the analysis, discussion of the results, and help in revision. All authors listed have made a substantial, direct and intellectual contribution to the work, and approved it for publication.

\section{ACKNOWLEDGMENTS}

The first author would like to acknowledge the Department of Science and Technology of the Government of India for providing financial support under the scheme of the INSPIRE Fellowship (IF170207) to carry out the present research.

Interdiscip J Nonlinear Sci. (2018) 28:063109. doi: 10.1063/1.5 026284

15. Bokhari A, Baleanu D, Belgacem R. Application of shehu transform to atangana-Baleanu derivatives. $J$ Math Computer Sci. (2020) 20:101-7. doi: 10.22436/jmcs.02 0.02 .03

16. Kumar S, Kumar A, Nieto JJ, Sharma B. Atangana-Baleanu Derivative With Fractional Order Applied to the Gas Dynamics Equations, Fractional Derivatives With Mittag-Leffler Kernel, Studies in Systems, Decision, and Control. Cham: Springer (2019). p. 194. doi: 10.1007/978-3-030-1166 2-0_14

17. Liu Y, Fan E, Yin B, Li H. Fast algorithm based on the novel approximation formula for the Caputo-Fabrizio fractional derivative. AIMS Mathe. (2020) 5:1729-44. doi: 10.3934/math.2020117

18. Jena RM, Chakraverty S, Rezazadeh H, Ganji DD. On the solution of time-fractional dynamical model of Brusselator reaction-diffusion system arising in chemical reactions. Mathe Methods Appl Sci. (2020) 43:111. doi: $10.1002 / \mathrm{mma} .6141$

19. Agarwal P, Baleanu D, Chen Y, Momani S, Machado JAT. Fractional Calculus: ICFDA, Springer Proceedings in Mathematics \& Statistics Book. Jordan: Springer (2018). p. 16-18. doi: 10.1007/978-981-15-0430-3

20. Jena RM, Chakraverty S, Baleanu D. A novel analytical technique for the solution of time-fractional Ivancevic option pricing model. Phys A: Statist Mech Appl. (2020) 550:124380. doi: 10.1016/j.physa.2020. 124380

21. Agarwal P. A Study of New Trends and Analysis of Special Function. Germany: LAP LAMBERT Academic Publishing (2013).

22. Jena RM, Chakraverty S, Yavuz M. Two-hybrid techniques coupled with an integral transformation for caputo time-fractional navier- stokes equations. Prog Fract Different App. (2020) 6:201-13. doi: 10.18576/pfda/ 060304

23. Chakraverty S, Jena RM, Jena SK. Time-fractional order biological systems with uncertain parameters. Morgan Claypool

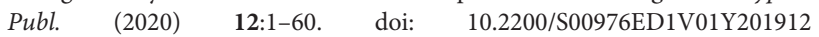
MAS031

24. Srivastava HM, Jena RM, Chakraverty S, Jena SK. Dynamic response analysis of fractionally-damped generalized bagley-torvik equation subject to external loads. Rus J Mathe Phys. (2020) 27:254-68. doi: 10.1134/S1061920820 020120

25. Agarwal P, Jain S, Mansour T. Further extended caputo fractional derivative operator and its applications. Rus J Mathe Phys. (2017) 24:41525. doi: 10.1134/S106192081704001X

26. Jena RM, Chakraverty S, Baleanu D. Solitary wave solution for a generalized hirota-Satsuma coupled $\mathrm{KdV}$ and MKdV equations: A semi-analytical approach. Alex Eng J. (2020). doi: 10.1016/j.aej.2020.01.002

27. Aboodh KS. Application of new transform aboodh transform to partial differential equations. Glob J Pure Appl Mathe. (2014) 10:249-54.

28. Aboodh KS. Solving fourth order parabolic PDE with variable coefficients using Aboodh transform homotopy perturbation method. Pure Appl Mathe J. (2015) 4:219-24. doi: 10.11648/j.pamj.20150405.13 
29. Zafar ZUA. Application of ZZ transform method on some fractional differential equations. Int J Adv Eng Global Technol. (2016) 4:1355-63.

30. Zafar ZUA. ZZ transform method. Int J Adv Eng Glob Technol. (2016) 4:1605-11.

31. Riabi L, Belghaba K, Cherif MH, Ziane D. Homotopy perturbation method combined with $\mathrm{ZZ}$ transform to solve some nonlinear fractional differential equations. Int J Anal App. (2019) 17:406-19. doi: 10.28924/2291-8639-17-2019-406

32. Aboodh KS, Idris A, Nuruddeen RI. On the aboodh transform connections with some famous integral transforms. Int J Eng Inform Syst. (2017) 1:143-51.
Conflict of Interest: The authors declare that the research was conducted in the absence of any commercial or financial relationships that could be construed as a potential conflict of interest.

Copyright (C) 2020 Jena, Chakraverty, Baleanu and Alqurashi. This is an open-access article distributed under the terms of the Creative Commons Attribution License (CC $B Y)$. The use, distribution or reproduction in other forums is permitted, provided the original author(s) and the copyright owner(s) are credited and that the original publication in this journal is cited, in accordance with accepted academic practice. No use, distribution or reproduction is permitted which does not comply with these terms. 